\title{
Polarization squeezing with cold atoms
}

\author{
V. Josse, A. Dantan, L. Vernac, A. Bramati, M. Pinard, and E. Giacobino \\ Laboratoire Kastler Brossel, Université Pierre et Marie Curie, Ecole Normale Supérieure et CNRS, \\ Case 74, 4 place Jussieu, 75252 Paris Cedex 05, France
}

(Dated: August 24, 2018)

\begin{abstract}
We study the interaction of a nearly resonant linearly polarized laser beam with a cloud of cold cesium atoms in a high finesse optical cavity. We show theoretically and experimentally that the cross-Kerr effect due to the saturation of the optical transition produces quadrature squeezing on both the mean field and the orthogonally polarized vacuum mode. An interpretation of this vacuum squeezing as polarization squeezing is given and a method for measuring quantum Stokes parameters for weak beams via a local oscillator is developed.
\end{abstract}

PACS numbers: 42.50.Dv, 42.50.Lc, 03.67.Hk

A great deal of attention has been recently given to the quantum features of the polarization states of the light, essentially because of their connections with quantum information technology. Several theoretical schemes to produce polarization squeezing using Kerr-like media have been proposed [1] and realized using optical fibers 2]. Other experimental realizations achieve polarization squeezing by mixing squeezed vacuum (generated by an OPO) with a strong coherent beam on a polarizing beam splitter [3] or mixing two independent quadrature squeezed beams (generated by an OPA) on a polarizing beam splitter [4]. Very recently it has been proposed to propagate a linearly polarized light beam through an atomic medium exhibiting self rotation to generate squeezed vacuum in the orthogonal polarization [5], which is equivalent to achieving polarization squeezing. In previous works [6] the interaction between a cloud of cold cesium atoms placed in a high finesse optical cavity and a circularly polarized laser beam nearly resonant with an atomic transition has been studied. Because of optical pumping, the atomic medium is conveniently modelled by an ensemble of two-level atoms. The saturation of the optical transition gives rise to an intensity-dependent refraction index. It is well known that the interaction of the light with a Kerr-like medium produces bistable behavior of the light transmitted by the cavity and that, at the turning point of the bistability curve, the quantum fluctuations of the light can be strongly modified and generate quadrature squeezing 7]. A noise reduction of $40 \%$ has thus been observed in our group 6 .

In this paper we focus on the theoretical and experimental investigation of polarization squeezing via the interaction of a linearly polarized laser beam with cold cesium atoms. In this configuration, the two-level atom model is no longer applicable and the situation much more complicated. We describe the interaction between light and the atomic medium by means of an $\mathrm{X}$-like four-level quantum model based on the linear input-output method. Our theoretical analysis shows clearly that competitive optical pumping may result in polarization switching, and polarization squeezing is predicted by the model [8]. In agreement with the model we observe quadrature squeezing in the probe laser mode and in the orthogonal vacuum mode. Experimentally, we obtain a polarization squeezing of $13 \%$ and we show for the first time that the quantum Stokes parameters for very weak beams can be measured together with their phases using a local oscillator (LO). In our case squeezing is due to cross-Kerr effect induced by the mean field rather than to the polarization self-rotation responsible for polarization switching.

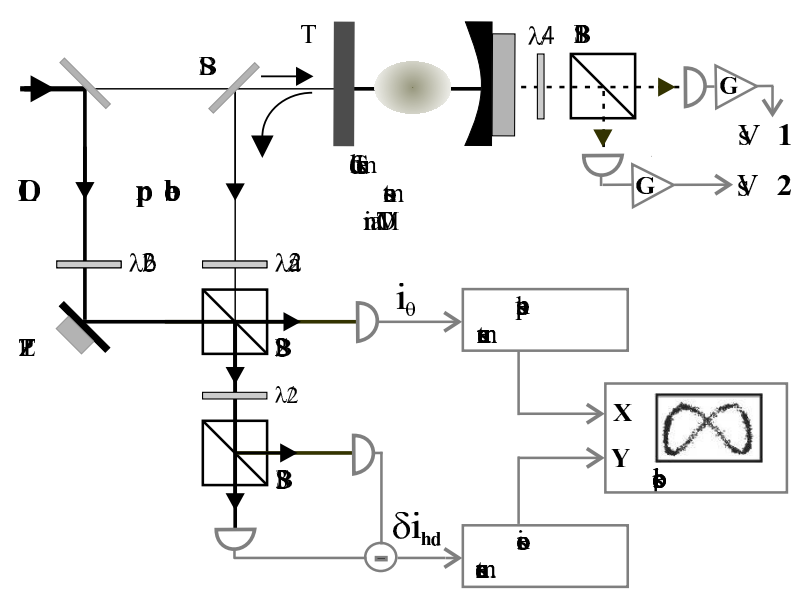

FIG. 1: Experimental set-up: PBS: polarizing beam splitter; BS: 10/90 beam splitter; $\lambda / 2$ : half-wave plate; PZT: piezoelectric ceramic.

The configuration used in the experiment is described in detail in [6]. In Fig. 1 we present the main features of the set-up. The cesium atoms are cooled in a standard magneto-optical trap which operates with three orthogonal circularly polarized trapping beam generated by a Ti:Sapphire laser and an inhomogeneous magnetic field. The trapping Ti:Sapphire laser is detuned by 3 times the linewidth of the upper state on the low frequency side of the $6 \mathrm{~S}_{1 / 2}, \mathrm{~F}=4$ to $6 \mathrm{P}_{3 / 2}, \mathrm{~F}=5$ transition. To prevent atoms from being optically pumped to the $6 \mathrm{~S}_{1 / 2}, \mathrm{~F}=3$ 
state, we superimpose a diode laser tuned to the $6 \mathrm{~S}_{1 / 2}$, $\mathrm{F}=3$ to $6 \mathrm{P}_{3 / 2}, \mathrm{~F}=4$ transition to the trapping beams. We use a $25 \mathrm{~cm}$ long linear cavity built around the cell. The cavity is close to the hemifocal configuration with a waist of $260 \mu \mathrm{m}$. The coupling mirror has a transmission coefficient $T$ of $10 \%$, the rear mirror is highly reflecting. Hence, the cavity is close to the bad cavity limit for which the cavity linewidth $(\kappa=5 \mathrm{MHz})$ is larger than the atomic linewidth $(\gamma=2.6 \mathrm{MHz})$. We probe the atoms with a linearly polarized laser beam detuned by about 50 $\mathrm{MHz}$ in the red of the $6 \mathrm{~S}_{1 / 2}, \mathrm{~F}=4$ to $6 \mathrm{P}_{3 / 2}, \mathrm{~F}=5$ transition. The optical power of the probe beam ranges from 5 to $15 \mu \mathrm{W}$. Under these conditions, the number of atoms interacting with the light beam is about $10^{6}-10^{7}$. The polarization of the light transmitted by the cavity is analyzed with a quarter-wave plate and a polarizing beamsplitter (PBS1), so that Vs1 and Vs2 give the amount of respectively left-handed $\left(I_{+}\right)$and right-handed $\left(I_{-}\right)$ circular light.

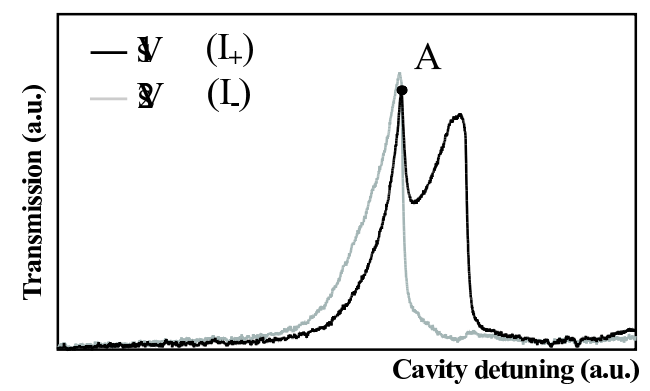

FIG. 2: Analysis of the circular polarization content of the light transmitted by the cavity and detected by the photodiodes shown in Fig. 1. Polarization switching occurs at point $A$. The power of the incident light is $7 \mu \mathrm{W}$.

A typical recording of the transmitted intensities $I_{ \pm}$as a function of the cavity length is shown in Fig. 2: starting from the left up to point $A$, the polarization remains linear (nearly equal amount of circular polarized light on both photodiodes), then it becomes circular. This polarization switching was known to occur in Fabry-Perot cavities containing atomic vapors with degenerate sublevels in the ground state [9, 10]. In the experiment the probe beam is nearly resonant with the $6 \mathrm{~S}_{1 / 2}, \mathrm{~F}=4$ to $6 \mathrm{P}_{3 / 2}, \mathrm{~F}=5$ transition: in principle, all the 20 Zeeman sub-levels are involved in the interaction. In order to get a qualitative physical insight into this problem, avoiding too heavy calculations, we model the atomic medium as an $X$-like four-level system [see Fig. 3].

The competitive optical pumping between the circular component $\sigma_{ \pm}$of light makes the linear polarization unstable inside the cavity above some intensity threshold. The optical pumping is responsible for polarization switching and the general shape of the cavity resonance curve [see Fig. 2] is well understood within this theoretical frame [8, 9, 10]. Alternatively, the polarization

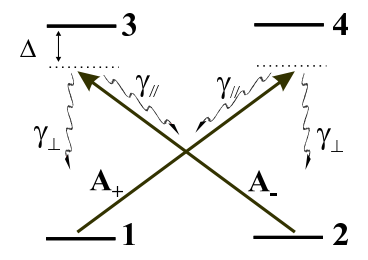

FIG. 3: Schematic energy level diagram for the X-like fourlevel system: $\gamma_{\perp}+\gamma_{\|}=\gamma$ is the optical dipole decay rate; $\Delta$ is the (large) detuning from resonance.

switching threshold may be interpreted as a laser oscillation threshold for the mode orthogonal to the main polarization mode [8]. Here, we are interested in the quantum fluctuations of the light, which can be strongly modified via the interaction with the atoms. When the polarization of the light is circular, the situation is analogous to the previous experimental scheme when the incoming field was circularly polarized [6]. We will therefore focus in the following on the case for which the polarization remains linear along the $x$-axis. The saturation of the $\sigma_{ \pm}$components of light causes the medium to behave as a Kerr-like medium for the mean field $\hat{A}_{x}$. In addition, the vacuum orthogonal $\hat{A}_{y}$ mode experiences a non linear dephasing via cross-Kerr effect [2, 8]. This system is then expected to generate quadrature squeezing for both modes; in the large detuning limit $[\Delta \gg \gamma]$, the equation for the vacuum mode fluctuations reads [8]

$$
\begin{aligned}
\frac{d \delta \hat{A}_{y}}{d t}= & -\left[\kappa+i\left(\Delta_{c}-\Delta_{0}\right)\right] \delta \hat{A}_{y} \\
& -i \Delta_{0} \frac{s_{x}}{2}\left[2 \delta \hat{A}_{y}-\frac{\left\langle\hat{A}_{x}\right\rangle^{2}}{\left|\left\langle\hat{A}_{x}\right\rangle\right|^{2}} \delta \hat{A}_{y}^{\dagger}\right]+\frac{2 \kappa}{\sqrt{T}} \delta \hat{A}_{y}^{i n}
\end{aligned}
$$

with $\Delta_{c}$ the cavity detuning, $\Delta_{0}=2 N g^{2} \kappa / \Delta T$ the linear atomic dephasing, $s_{x}=2 g^{2}\left|\left\langle\hat{A}_{x}\right\rangle\right|^{2} / \Delta^{2}$ the saturation parameter and $\delta \hat{A}_{y}^{i n}$ the incident field fluctuations. The cross-Kerr induced term has two contributions: a dephasing $\left(\propto \mid\left\langle\hat{A}_{x}\right\rangle^{2} \delta \hat{A}_{y}\right)$ and the term in $\left\langle\hat{A}_{x}\right\rangle^{2} \delta \hat{A}_{y}^{\dagger}$, responsible for the squeezing of this mode. A similar equation can be derived for the mean field $\hat{A}_{x}$, for which the squeezing is then generated via the usual Kerr term $\left(\propto\left\langle\hat{A}_{x}\right\rangle^{2} \delta \hat{A}_{x}^{\dagger}\right)$. These equations are valid when the large excess noise due to optical pumping can be neglected, that is for times smaller than the optical pumping time, in contrast with [5]. Squeezing is then predicted for noise frequencies higher than the inverse optical pumping time. Using the experimental set-up described in Fig. 1, we have measured the quadrature noise of both modes. The signal recorded at the output of the cavity (see Fig. 2) is used to lock the cavity length on the regime where the polarization remains linear. After interacting with the atoms, both fields are mainly reflected by BS and then mixed on PBS2 with a $10 \mathrm{~mW}$ local oscillator beam (LO). Using the half-wave plate $\lambda / 2 a$, we are able to send either 
the mean field mode or the orthogonal vacuum mode to PBS3 and perform the usual homodyne detection. By varying the relative phase of the $\mathrm{LO}$ with respect to the probe beam we can detect the noise features of all the quadratures of the field. In agreement with the theoretical predictions, we observe quadrature squeezing on both the main mode and the orthogonal mode. The results are $5 \%$ (7\% after correction for optical losses, mainly due to BS) of noise reduction for the main polarization mode at $6 \mathrm{MHz}$ and $13 \%$ (18\% after correction) for the squeezed vacuum state at $3 \mathrm{MHz}$, as shown in Fig. 4. This system is then able to produce simultaneously two squeezed modes, for which the relatives phases are intrinsically fixed. Let us note that these two squeezed modes can be used to generate a pair of entangled beams, which will be presented in a forthcoming publication [11].

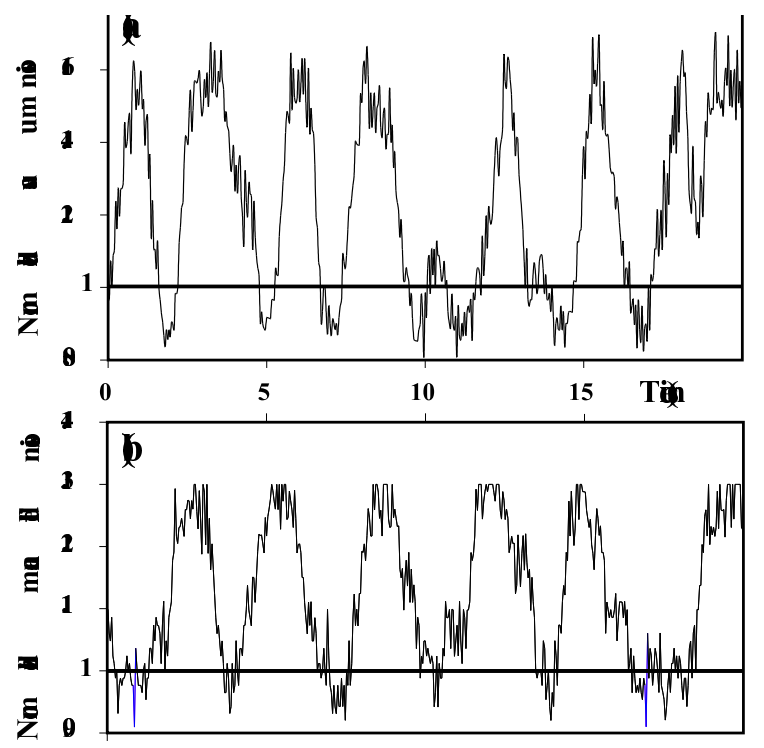

FIG. 4: Normalized quadrature noise for the vacuum field mode at $3 \mathrm{MHz}$ (a) and for the mean field mode at $6 \mathrm{MHz}$ (b). The best squeezing is $13 \%$ for the vacuum mode and $5 \%$ for the mean field mode.

In the following we focus on the study of the noise of the mode with orthogonal polarization with respect to the main mode, commonly referred to as polarization noise. The characterization of the quantum features of the polarization state relies on the measurement of the quantum Stokes parameters [12]. They are defined from their classical counterparts

$$
\begin{array}{ll}
\hat{S}_{0}=\hat{A}_{x}^{\dagger} \hat{A}_{x}+\hat{A}_{y}^{\dagger} \hat{A}_{y}, & \hat{S}_{1}=\hat{A}_{x}^{\dagger} \hat{A}_{x}-\hat{A}_{y}^{\dagger} \hat{A}_{y} \\
\hat{S}_{2}=\hat{A}_{x}^{\dagger} \hat{A}_{y}+\hat{A}_{y}^{\dagger} \hat{A}_{x}, & \hat{S}_{3}=i\left(\hat{A}_{y}^{\dagger} \hat{A}_{x}-\hat{A}_{x}^{\dagger} \hat{A}_{y}\right)
\end{array}
$$

These operators obey the commutation relations $\left[\hat{S}_{0}, \hat{S}_{i}\right]=0$ and $\left[\hat{S}_{i}, \hat{S}_{j}\right]=\epsilon_{i j k} 2 i \hat{S}_{k}(i=1,2,3)$. Their spectral noise densities satisfy uncertainty relations $V_{\hat{S}_{i}}(\omega) V_{\hat{S}_{j}}(\omega) \geq\left|\epsilon_{i j k}\left\langle\hat{S}_{k}\right\rangle\right|^{2}$. In our case the light is lin- early polarized along the $x$-axis, then $\left\langle\hat{S}_{0}\right\rangle=\left\langle\hat{S}_{1}\right\rangle=\alpha_{x}^{2}$ and $\left\langle\hat{S}_{2}\right\rangle=\left\langle\hat{S}_{3}\right\rangle=0$, where $\left\langle\hat{A}_{x}\right\rangle=\alpha_{x}$ is chosen real. Then the only non trivial Heisenberg inequality is $V_{\hat{S}_{2}}(\omega) V_{\hat{S}_{3}}(\omega) \geq \alpha_{x}^{4}$. Polarization squeezing is then achieved if $V_{\hat{S}_{2}}$ or $V_{\hat{S}_{3}}$ is below the coherent state value $\alpha_{x}^{2}$. The fluctuations of $\hat{S}_{2}$ and $\hat{S}_{3}$ are related to the fluctuations of the quadratures of the vacuum orthogonal mode

$$
\delta \hat{S}_{2}=\alpha_{x}\left(\delta \hat{A}_{y}^{\dagger}+\delta \hat{A}_{y}\right), \quad \delta \hat{S}_{3}=i \alpha_{x}\left(\delta \hat{A}_{y}^{\dagger}-\delta \hat{A}_{y}\right)
$$

The physical meaning of the Stokes parameters fluctuations is the following: the $\delta \hat{S}_{2}$ fluctuations lead to a geometric jitter of the polarization axis, whereas the $\delta \hat{S}_{3}$ fluctuations are linked to ellipticity fluctuations. It can be seen from Eq. (2) that these fluctuations are related to the amplitude and phase fluctuations of $\hat{A}_{y}$. Therefore polarization squeezing is equivalent to vacuum squeezing on the orthogonal mode.

The measurement of the Stokes parameters can be carried out directly by means of two balanced photodiodes and suitable combinations of half-wave and quarter-wave plates [12]. In our set-up, however, the power of the probe beam interacting with the atoms $(\sim 10 \mu \mathrm{W})$ is not sufficient, so that we need a LO for the detection. The fluctuations of the vacuum mode $\hat{A}_{y}$ are measured using the homodyne detection described above. Following Eq. (2) the photocurrent can be expressed in terms of the fluctuations of $\hat{S}_{2}$ and $\hat{S}_{3}$ :

$$
\delta i_{h d} \propto \cos \theta_{h d} \delta \hat{S}_{2}+\sin \theta_{h d} \delta \hat{S}_{3} \equiv \delta \hat{S}_{\theta_{h d}}
$$

where $\theta_{h d}$ is the relative phase between the $\mathrm{LO}$ and the mean field. As $\theta_{h d}$ is varied in time, we correspondingly detect the fluctuations of the Stokes parameter $\hat{S}_{\theta_{h d}}$. For instance, $\theta_{h d}=0$ (respectively $\pi / 2$ ) corresponds to the detection of the fluctuations of $\hat{S}_{2}$ (respectively of $\hat{S}_{3}$ ). Hence, in the experiment we can get the Stokes parameters simply by simultaneously measuring the relative phase $\theta_{h d}$ and the quadrature noise of the vacuum mode. This measurement is readily performed by setting the half-wave plate before PBS2 in such a way that the $\hat{A}_{y}$ mode is sent to the homodyne detection; the mean field $\hat{A}_{x}$ goes through the other port of the beam splitter and is detected together with a portion of the LO by a photodiode (see Fig. 1). The phase is determined via the interference signal between LO and $\hat{A}_{x}\left(i_{\theta} \propto \cos \theta_{h d}\right)$. The two signals $i_{\theta}$ and $\delta i_{h d}$ are sent to the $X Y$ channel of the oscilloscope, giving the characteristic curves reported below.

In Fig. 5 the normalized quadrature noise of $\hat{A}_{y}$, obtained at a noise frequency of $3 \mathrm{MHz}$, is plotted as a function of the relative phase between the mean field and the LO. In agreement with Eq. (3), it can be seen that the noise of $\hat{S}_{2}$ is given by the extreme 

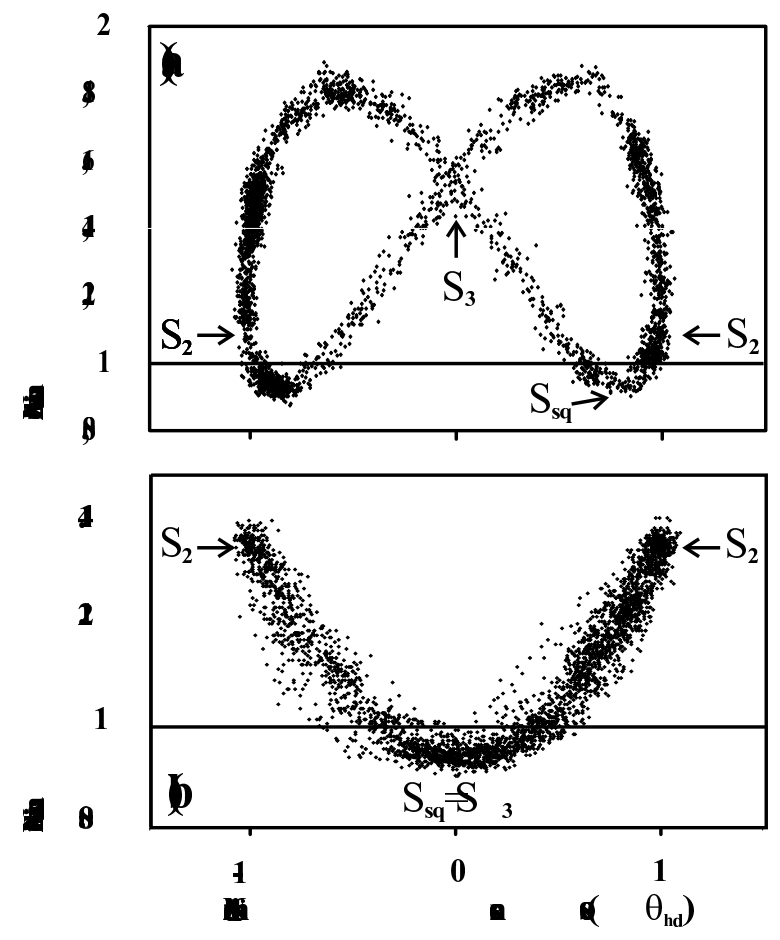

FIG. 5: Normalized quadrature noise at $3 \mathrm{MHz}$ for the vacuum mode $\hat{A}_{y}$ vs the normalized interference signal: $\cos \theta_{h d}$. The general case is shown in curve (a): polarization squeezing is achieved when $\theta_{h d}=\theta_{s q}= \pm 30^{\circ}$ : a linear combination of $\hat{S}_{2}$ and $\hat{S}_{3}$ is squeezed. In curve (b), $\hat{S}_{3}$ is squeezed $\left(\theta_{s q}= \pm \pi / 2\right)$.

points $\theta_{h d}=0, \pm \pi$ on the diagram and that of $\hat{S}_{3}$ by the center point $\theta_{h d}= \pm \pi / 2$. In general, for an arbitrary squeezed quadrature, a linear combination of $\hat{S}_{2}$ and $\hat{S}_{3}$ is squeezed (Fig. 5a). We find that the polarization squeezing strongly depends on the operating point and on the noise frequency. For instance, in Fig. 5b, we see that $\hat{S}_{3}$ is squeezed.

To conclude, we have demonstrated that the nearly resonant interaction of a linearly polarized laser beam with a cloud of cold atoms in an optical cavity can produce quadrature squeezing on the mean field mode and on the orthogonally polarized vacuum mode. We have shown that these results can be interpreted as polariza- tion squeezing and developed a method to measure the quantum Stokes parameters for weak beams, using a local oscillator and a standard homodyne detection.

This work was supported by the QIPC European Project No. IST-1999-13071 (QUICOV).

[1] A.S. Chirkin, A.A. Orlov, D.Yu. Paraschuk, Quantum Electron. 23, 870 (1993); A.P. Alodjants, A.M. Arakelian, A.S. Chirkin, JEPT 108, 63 (1995); N.V. Korolkova, A.S. Chirkin, J. Mod. Opt. 43, 869 (1996).

[2] L. Boivin, H.A. Haus, Optics Lett. 21, 146 (1996); J. Heersink, T. Gaber, S. Lorenz, O. Glöckl, N. Korolkova, G. Leuchs, quant-ph/0302100

[3] P. Grangier, R.E. Slusher, B.Yurke, A. LaPorta, Phys. Rev. Lett. 59, 2153 (1987); J. Hald, J.L. Sorensen, C. Schori, E.S. Polzik, Phys. Rev. Lett. 83, 1319 (1999).

[4] W.P. Bowen, R. Schnabel, H.A. Bachor, P.K. Lam, Phys. Rev. Lett. 88, 093601 (2002).

[5] A.B. Matsko, I. Novikova, G.R. Welch, D. Budker, D.F. Kimball, S.M. Rochester, Phys. Rev. A 66, 043815/1 (2002); I. Novikova, A.B. Matsko, G.R. Welch, private communication.

[6] A. Lambrecht, E. Giacobino, J.M. Courty, Optics Comm. 115, 199, (1995); A. Lambrecht, T. Coudreau, A.M. Steimberg, E. Giacobino, Europhys. Lett. 36, 93 (1996); A.Z. Khoury, T. Coudreau, C. Fabre, E. Giacobino, Phys. Rev. A 57, 4397 (1998).

[7] L. Hilico, C. Fabre, S. Reynaud, E. Giacobino, Phys. Rev. A 46, 4397 (1992).

[8] V. Josse, A. Dantan, A. Bramati, M. Pinard, E. Giacobino, to appear in J. Opt. B, Special Issue on Quantum optics and Quantum entanglement, quant-ph/0302142

[9] E. Giacobino, Optics Comm. 56, 249 (1985).

[10] S. Cecchi, G. Giusfredi, E. Petriella, P. Salieri, Phys. Rev. Lett. 49, 1928 (1982); M.W. Hamilton, W.J. Sandle, J.T. Chilwell, J.S. Satchell, D.M. Warrington, Optics Comm. 48, 190 (1983).

[11] V. Josse, A. Dantan, A. Bramati, M. Pinard, E. Giacobino, to be published.

[12] N. Korolkova, G. Leuchs, R. Loudon, T.C. Ralph, C. Silberhorn, Phys. Rev. A, 65, 052306 (2002); N. Korolkova, Ch. Silberhorn, O. Glöckl, S. Lorenz, Ch. Marquardt, G. Leuchs, Eur. Phys. J. D, 18, 229 (2002). 